\title{
Reaproveitamento de resíduos sólidos para confecção de blocos de concreto
}

\author{
Reuse of solid wast to make concrete blocks \\ Pedro Augusto Paiva de Carvalho ${ }^{\dagger *}$, Luiz Gustavo da Silva de Lima Moreira ${ }^{\dagger}$, Barbara Braga \\ Barbosa
}

Como citar esse artigo. de

Carvalho, PAP; Moreira, LGSL; Barbosa, BB. Reaproveitamento de resíduos sólidos para confecção de blocos de concreto. Revista Teccen. 2019 Jul/Dez; 12 (2): 102-108

\begin{abstract}
Resumo
Os resíduos sólidos envolvem uma série de materiais que são descartados, muitas vezes, em locais inadequados, sendo que um dos principais produtores desses elementos é a construção civil, o que leva à necessária reflexão sobre o tema, visando buscar meios de minimizar esse problema que gera impactos ambientais sérios, além de prejuízos nos canteiros de obras, em face do desperdício ocorrido. O gerenciamento dos resíduos sólidos da construção civil é aspecto marcante relacionado ao tema, pois é preciso que ocorra um processo de planejamento e administração de todo o volume de resíduos, para que recebam a devida destinação, a partir de uma seleção de materiais de forma a identificar os que podem ou não ser reaproveitados e assim, seja possível determinar o melhor a ser feito com os mesmos. Neste sentido, é preciso que se pense em reaproveitamento dos resíduos sólidos e para o presente estudo, focou-se esse processo focando a confecção de blocos de concreto, que podem ser reutilizados na própria obra, gerando benefícios não apenas ambientais, como também econômicos. A metodologia do estudo é qualitativa, com pesquisa bibliográfica e o objetivo é justamente refletir sobre o reaproveitamento de resíduos sólidos para confecção de blocos de concreto.

Palavras-Chave: Resíduos Sólidos; Construção Civil; Blocos de Concreto.
\end{abstract}

\begin{abstract}
Solid waste involves a series of materials that are often disposed of in inappropriate places, and one of the main producers of these elements is construction, which leads to the necessary reflection on the subject, seeking ways to minimize this problem that generates serious environmental impacts, as well as damage to construction sites due to waste. The management of solid waste from civil construction is a striking aspect related to the theme, since it is necessary to have a process of planning and administration of the entire volume of waste, so that they are properly disposed of, from a selection of materials in order to identify those that may or may not be reused and thus determine the best that can be done with them. In this sense, it is necessary to think about the solid waste reuse and for the present study, this process focused on the making of concrete blocks, which can be reused in the work itself, generating not only environmental but also economic benefits. The methodology of the study is qualitative, with bibliographic research and the objective is to reflect on the reuse of solid waste to make concrete blocks.

Keywords: Solid Waste; Construction; Concrete blocks.
\end{abstract}

\section{Introdução}

O crescimento indiscriminado da população urbana fez com que o volume de resíduos sólidos aumentasse significativamente, gerando um sério problema para a sociedade, visto que não há lugares adequados suficientes para comportar todo o lixo produzido pelos cidadãos brasileiros. Esse problema se intensificou significativamente, através do grande volume de entulhos produzido pelas ações da construção civil, revelando a necessidade de se demandar uma atenção maior ao tema.

Os resíduos sólidos tem origem a partir das atividades humanas e em face do crescimento desordenado das cidades, além do aumento das atividades da construção civil para atender a demanda, é possível observar um grande volume de entulhos, que muitas vezes não recebe a destinação final adequada, já que faltam locais adequados, valendo destacar que

Afiliação dos autores: † Graduando em Engenharia Civil pela Universidade de Vassouras;

$\$$ MSc. em Engenharia Ambiental e Docente pela Universidade de Vassouras.

* Email para correspondência: pedroaugustoeng@hotmail.com 
muitos desses materiais podem ser reutilizados, o que motiva benefícios não apenas para o meio ambiente, mas também para os responsáveis pelas obras.

Foram criadas normas e até políticas públicas justamente para nortear as ações adequadas para um processo de gerenciamento dos resíduos sólidos, inclusive gerados pela construção civil, mas o que se observa na realidade, é a falta de planejamento e aplicação das mesmas, de modo que esses materiais acabam sendo destinados a áreas inadequadas, agravando cada vez mais, os impactos ao meio ambiente.

As políticas públicas são instrumentos utilizados pelos entes públicos para intervenção do Estado na sociedade, visando manter o equilíbrio da mesma, especialmente na esfera ambiental, como forma de aplicar alguns mecanismos indispensáveis para preservação dos recursos naturais.

Dentre os inúmeros resíduos produzidos, os gerados pela construção civil são os que revelam maior volume e geram inúmeros prejuízos ao meio ambiente, o que tem motivado maior preocupação por parte da sociedade em relação a esse tipo de resíduo, que impactam o planeta a partir do momento que gera desequilíbrios ambientais, colaborando para o surgimento de eventos naturais, além de aumentar os custos das obras, em face do desperdício.

Para minimizar os problemas gerados pelos resíduos sólidos da construção civil é importante que se busquem alternativas viáveis, como a recuperação e reutilização desses resíduos, promovendo-se a reciclagem para que reduza a quantidade de recursos naturais retirados para a produção de novos produtos e também do volume de lixo descartado.

Neste contexto, muitos materiais que compõem os resíduos sólidos da construção civil podem ser reaproveitados, inclusive para confecção de materiais que podem ser utilizados nas próprias obras, reduzindo os custos das mesmas, como é o caso dos blocos de concreto, que podem ser produzidos a partir de materiais recicláveis.

Frente a todo o exposto, desenvolveu-se um estudo sobre o tema "reaproveitamento de resíduos sólidos para elaboração de blocos de concreto", sendo adotada a metodologia qualitativa predominante, com uma pesquisa bibliográfica, que contou com várias fontes encontradas na literatura pátria.

O objetivo do estudo é refletir sobre o reaproveitamento de resíduos sólidos para confecção de blocos de concreto.

O desenvolvimento do estudo foi organizado em três tópicos distintos, a saber: Resíduos sólidos da construção civil; Gerenciamento dos resíduos gerados na construção civil; Reaproveitamento dos resíduos sólidos para confecção de blocos de concreto.

\section{Metodologia}

A metodologia de pesquisa adotada para o desenvolvimento deste estudo foi de caráter qualitativo, com predominância de uma pesquisa bibliográfica, que teve como fontes de pesquisa artigos, trabalhos de conclusão de curso, teses, livros e outras publicações físicas ou digitalizadas, estas últimas retiradas do Google acadêmico, Scielo e Lilacs.

Segundo Araújo e Gouveia (2018, p. 4):

Pesquisa Bibliográfica: este tipo de pesquisa é elaborada a partir de material já publicado, livros, revistas, artigos e material disponibilizado na Internet, entre outras fontes. Este tipo de pesquisa explica um problema a partir de referenciais teóricos publicados em documentos. Pode ser realizada independentemente ou como parte da pesquisa descritiva ou experimental. Ambos casos buscam conhecer e analisar as contribuições culturais ou científicas do passado, existentes sobre um determinado assunto, tema ou problema.

Para a seleção das fontes de pesquisa foram destacados os seguintes termos e expressões "resíduos sólidos", "reaproveitamento de resíduos sólidos", "resíduos sólidos da construção civil", "reaproveitamento dos resíduos sólidos para confecção de blocos de concreto". Além disso, foi determinado como recorte temporal um recorte temporal do período de 2002 até 2019, sendo preferenciais as referências mais atualizadas, mas destacando-se também legislações relevantes ao tema, que datam períodos mais antigos.

\section{Fundamentação Teórica}

\section{Resíduos sólidos da construção civil}

Os resíduos sólidos resultam das atividades humanas, especialmente em áreas urbanas, já que nelas há grande concentração de pessoas e o volume dos resíduos produzidos pelas mesmas é maior, tornandose um empecilho para a sociedade e revelando a necessidade dos entes públicos disponibilizarem locais adequados para seu despejo.

O problema é que o grande volume e a falta de tratamento dos resíduos sólidos antes de seu despejo direto no solo tem gerado intensa poluição em determinadas áreas, o que gera grande preocupação não só da sociedade, mas também do Estado.

Para que seja possível melhor compreender o termo resíduos sólidos, cabe apresentar sua definição, partindo-se do disposto na Lei 12.305/2010 que instituiu a chamada Política Nacional de Resíduos Sólidos, regulamentando aspectos fundamentais como sua separação, destinação, gerenciamento e demais esclarecimentos para minimizar os efeitos prejudiciais dos mesmos no meio ambiente, quando de sua geração 
e gestão inadequada (Brasil, 2010).

Segundo a Política Nacional de Resíduos Sólidos, resíduos sólidos são definidos como:

[...] material, substância, objeto ou bem descartado resultante de atividades humanas em sociedade, a cuja destinação final se procede, se propõe proceder ou se está obrigado a proceder, nos estados sólido ou semissólido, bem como gases contidos em recipientes e líquidos cujas particularidades tornem inviável o seu lançamento na rede pública de esgotos ou em corpos d'água, ou exijam para isso soluções técnica ou economicamente inviáveis em face da melhor tecnologia disponível (Brasil, 2010, p. 2).
Nota-se que todo e qualquer tipo de material originário de ações humanas no ambiente social, que é descartado em áreas que não sejam esgotos e corpos d'água, demandando um cuidado, gerenciamento e adequada destinação, desde que no estado sólido ou semissólido ou outros estados mantidos em recipientes, é considerado como resíduo sólido.

É válido observar que há uma grande preocupação relacionada à questão dos resíduos sólidos, isso devido ao enorme volume dos mesmos que é produzido diariamente no Brasil, conforme se pode observar através da Figura 1, a saber:

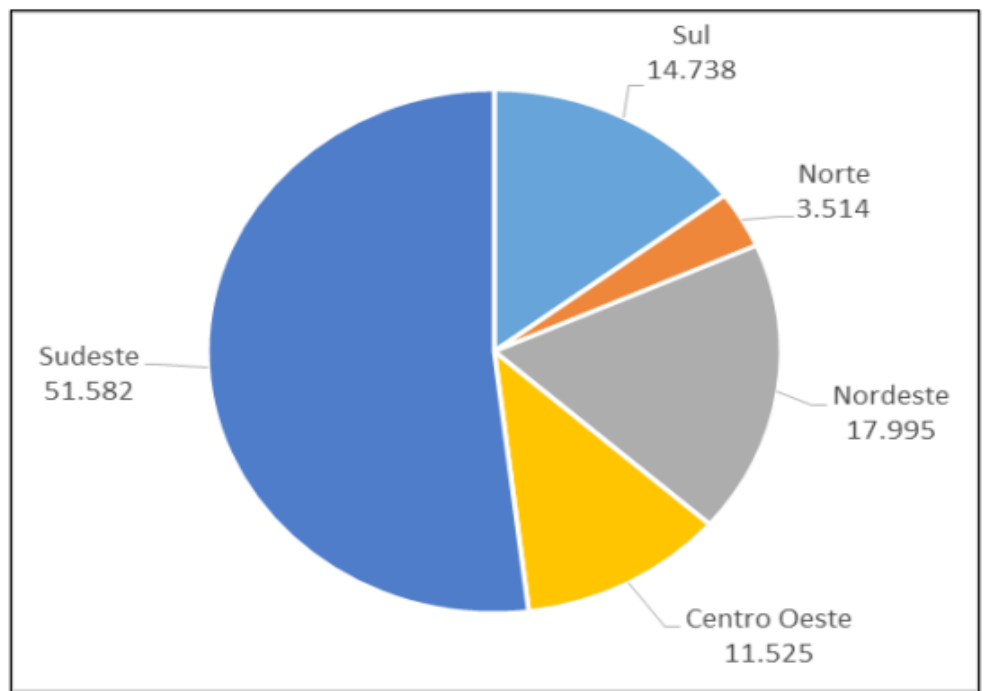

Figura 1. Estimativa do volume diário de resíduos sólidos/região do Brasil (t) Fonte: Marcetti e Lírio (2018, p. 24).

A Figura 1 revela uma estimativa do volume diário de resíduos sólidos gerados em cada região brasileira, sendo possível observar que o Sudeste é a região que mais produz esses materiais, chegando a 51.582 toneladas, o que demanda atenção e a necessidade de aplicações de políticas públicas para minimizar os problemas gerados por esse montante, especialmente no contexto ambiental.

No âmbito da construção civil, os resíduos sólidos gerados são conceituados como entulhos, ou seja, representam os rejeitos de materiais variados que tem origem a partir da realização de obras como reparo, construções, demolições, restaurações, entre outras, o que gera um elevado volume, sendo imprescindível que seja dada a devida destinação aos mesmos (Fernandes, 2018).

Garcia (2018) observa que no Brasil, cerca de $53 \%$ de todo o resíduo sólido produzido é constituído de entulhos da construção civil, além disso, grande parte dos mesmos são dispostos em locais inadequados, em face da falta de gerenciamento adequado, o que fere as normas vigentes no país.

Leite et. al. (2018) observam que os resíduos sólidos oriundos da construção civil, comumente conhecidos como entulhos, representam sobres de material utilizado em obras e quaisquer ações realizadas pela área de construção civil, englobando uma série de fragmentos que, em grande parte, podem ser reaproveitados com o devido planejamento e gerenciamento dos mesmos.

Frise-se que a construção civil exerce um papel de suma importância na esfera nacional, influenciando muito no Produto Interno Bruto (PIB) do país, o que revela a necessidade de suas ações, mas é fundamental que estas sejam planejadas e apresentem programas voltados para a preservação do meio ambiente, especialmente através de ações que minimizem os efeitos negativos do grande volume de resíduos sólidos gerados através das atividades da construção civil (Leite et al., 2018).

Um importante aspecto sobre o tema é apresentado pela Resolução n. 307/2002 do CONAMA, 
que atribui aos municípios a responsabilidade de descarte dos resíduos da construção civil, acrescentando o seguinte texto:

[...] as responsabilidades e os deveres de cada município no sentido de licenciar as áreas para disposição final de resíduos da construção civil. Para isso, os municípios devem implementar o Plano Integrado de Gerenciamento de Resíduos da Construção Civil, semelhante ao Plano de Gerenciamento de Resíduos Sólidos (PGSR) (Brasil, 2002).

Conforme dispõe o referido dispositivo legal, é recomendado que não sejam gerados resíduos e quando não houver outra alternativa, eles devem receber uma destinação adequada, visando dessa forma, minimizar os prejuízos ao meio ambiente (Fernandes, 2018).

É relevante esclarecer que a construção civil é responsável por até cinquenta por cento do uso de recursos naturais, gerando elevado volume de entulhos ou restos de materiais que são depositados em lugares que não são adequados para serem jogados os resíduos de construção, causando dessa forma, impactos ambientais muito relevantes no mundo inteiro (Fernandes, 2018).

\section{Gerenciamento dos resíduos gerados na construção civil}

É importante entender que o gerenciamento de resíduos sólidos gerados através das atividades da construção civil representa um processo complexo, que envolve a criação de uma política de gestão ambiental, implementada a partir de um processo reverso, com objetivo de promover a redução do volume desses materiais, através do estímulo à conscientização de pessoas que atuam em canteiros de obras (Leite et al., 2018).

Através do gerenciamento dos resíduos da construção civil, é possível selecionar os materiais que podem ou não ser reaproveitados, podendo-se oferecer a destinação mais adequada a cada um deles, de modo a reduzir os impactos que os mesmos geram ao meio ambiente, assim como nos custos da produção e para tanto, é preciso reciclar o maior volume possível.

É importante entender que o gerenciamento dos resíduos sólidos, leva em conta a classificação dos mesmos, que de acordo com a NBR 10004/04 varia de acordo com as características do material que o compõe, podendo ser denominado como de classe I, quando se tratam de elementos perigosos; de classe II, quando forem não inertes e, por fim, Classe III, englobando os materiais inerentes, valendo destacar que esses últimos abrangem os entulhos produzidos pela construção civil (Zanandréa, 2018).

Zanandréa(2018) destaca ainda outra classificação para os resíduos sólidos, consubstanciada na Resolução n. 307/2002 da CONAMA, que os divide em Classe A, quando forem reutilizáveis, havendo possibilidade de reciclagem como agregados; Classe $\mathrm{B}$, referindo-se aos materiais reutilizados para destinações distintas; Classe $\mathrm{C}$, remetendo aos materiais que não receberam tecnologia necessária para sua reciclagem e, por fim, Classe D, quando os resíduos forem perigosos.

A classificação dos resíduos sólidos de origem da construção civil é aspecto essencial para promoção de seu gerenciamento, isso porque é preciso que ocorra todo um processo de separação dos diferentes materiais, visando dessa maneira, identificar quais deles podem ser reaproveitados; em seguida, é necessário que ocorra o acondicionamento adequado dos mesmos, considerando suas respectivas classes; em um terceiro momento, é preciso armazena-los, para só então, iniciar uma quarta fase da reutilização dos materiais que podem ser reciclados, visando dessa forma, gerar lucro através do reuso além de reduzir, significativamente, o volume dos resíduos destinados aos aterros sanitários, que tanto impactam o meio ambiente (Silva et al. 2018).

Independentemente do tipo de material gerado na construção civil, o fato é que irá gerar prejuízos ao meio ambiente, sendo fundamental a realização de ações que tentem minimizá-los e através do gerenciamento dos resíduos, é possível determinar a destinação final mais adequada a cada tipo de material, conforme sua classificação, mitigando o impacto ambiental.

Dantas (2018) observa que o gerenciamento dos resíduos sólidos da construção civil precisa ocorrer de forma integrada, sendo fundamental a aplicação do chamado Princípio dos 3 R'S, ou seja, com aplicação de ações bem planejadas que envolvam redução, reutilização e reciclagem dos materiais, como objetivo principal para minimizar prejuízos ambientais gerados pela má destinação desses resíduos.

Segundo Dantas (2018, p. 4):

Reduzir significa consumir menos produtos e preferir aqueles que ofereçam menor potencial de geração de resíduos e tenham maior durabilidade enfatizando o combate ao desperdício, diminuindo a quantidade de resíduo sólido gerado. - Reutilizar significa utilizar outras vezes, sem transformação, para outras finalidades um material já foi originalmente usado, como por exemplo uma embalagem, reinserindo-o no ciclo produtivo. Reciclar é transformar o resíduo antes inútil em matérias-primas ou novos produtos, ou seja, é um processo de reinserção de um determinado produto como matéria-prima na manufatura de bens.

Ainda com base nos estudos de Dantas (2018), é válido considerar que na atualidade, há alguns estudos que já trazem o Princípio em questão acrescido de mais dois elementos, ou seja, repensar e recusar, visando dessa maneira estimular um processo de conscientização que leve as pessoas a garantir uma redução relevante no volume dos resíduos sólidos.

Pontes e Araújo (2018) entendem que ocorrendo o planejamento e aperfeiçoamento do gerenciamento 
dos resíduos sólidos da construção civil, é possível que ela possa absorver grande parte dos materiais gerados em suas obras, de modo a reaproveita-los de diferentes maneiras, das quais, destaca-se a confecção de blocos de concreto, tema tratado no tópico a seguir.

\section{Reaproveitamento dos resíduos sólidos para confecção de blocos de concreto}

Em face do elevado volume de resíduos sólidos gerados pela construção civil, é preciso buscar alternativas viáveis para reduzir os problemas que ele causa, surgindo neste contexto, a ideia do reaproveitamento dos entulhos oriundos da construção civil, o que traz inúmeros benefícios, sejam eles econômicos ou ambientais, em face da redução de custos de gerenciamento, valor agregado do que é reciclado ser muitas vezes menor que o agregado natural, entre outros fatores que motivam um processo de reaproveitamento (Dantas, 2018).

Para que se tenha uma ideia da dimensão do problema, Medrade (2018) explica que a construção civil é responsável por uma produção de aproximadamente $50 \%$ de todo o resíduo sólido gerado no Brasil e grande parte desse material é destinado indevidamente, ocasionando prejuízos significativos, especialmente em face do desperdício, já que parte desses resíduos pode ser reaproveitado, o que além de contribuir para o meio ambiente, ainda gera economia para toda a obra, principalmente quando esses elementos retornam para a construção civil, tendo utilidade específica.

Silva (2019) enfatiza a necessidade de se reaproveitar resíduos sólidos da construção civil, para reduzir a retirada de matérias-primas do ambiente natural destinados à confecção de blocos de concreto, minimizando assim, o impacto ambiental, sendo necessária a seleção de materiais residuais que apresentem maior resistência, visando dessa maneira, ter como resultado blocos de melhor qualidade, que atendam às necessidades das obras.

Silva (2019) destaca a questão da resistência enquanto aspecto marcante, uma vez que, o reaproveitamento de resíduos sólidos da construção civil para a confecção de blocos de concreto, não pode gerar um produto inferior e frágil, sendo fundamental que a resistência seja considerada, para garantir maior segurança à obra e para isso, é notável a relevância de se considerar alguns requisitos básicos, apresentados na Tabela 1.

Tabela 1. Resistência característica a compressão de blocos de concretos.

\begin{tabular}{|c|c|c|c|c|c|c|c|}
\hline \multirow{3}{*}{ CLASSIFICAÇÃO } & \multirow{3}{*}{ CLASSE } & \multirow{3}{*}{$\begin{array}{c}\text { RESISTÊNCIA } \\
\text { CARACTERISTICA A } \\
\text { COMPRESSÃO AXIAL (Mpa) }\end{array}$} & \multicolumn{4}{|c|}{ ABSORÇÃO (\%) } & \multirow{3}{*}{$\begin{array}{c}\text { RETRAÇÃO } \\
(\%)\end{array}$} \\
\hline & & & \multicolumn{2}{|c|}{ NORMAL } & \multicolumn{2}{|c|}{ AGREGADO LEVE } & \\
\hline & & & INDIVIDUAL & MÉDIA & INDIVIDUAL & MÉDIA & \\
\hline \multirow{2}{*}{$\begin{array}{c}\text { Com Função } \\
\text { estrutural }\end{array}$} & A & $F b k \geq 8,0$ & $\leq 9,0$ & $\leq 8,0$ & \multirow{3}{*}{$\leq 16,0$} & \multirow{3}{*}{$\leq 13,0$} & \multirow{3}{*}{$\leq 0,065$} \\
\hline & $\mathrm{B}$ & $4,0 \leq F b k<8,0$ & $\leq 10,0$ & $\leq 9,0$ & & & \\
\hline $\begin{array}{c}\text { Com ou sem função } \\
\text { estrtural }\end{array}$ & $\mathrm{C}$ & $F b k \geq 3,0$ & $\leq 11,0$ & $\leq 10,0$ & & & \\
\hline
\end{tabular}

É fundamental que as características apresentadas na Tabela 1 sejam consideradas para a verificação da resistência dos blocos de concreto confeccionados com materiais reaproveitados, valendo destacar o resultado do ensaio de resistência à compressão, apresentado nos estudos de Silva (2019), que permitiram concluir que o uso de elementos como o pó de vidro temperado agregado na confecção dos blocos de concreto, gera um produto final satisfatório,atendendo aos requisitos elencados na tabela supramencionada, o que revela que esse tipo de material não compromete a qualidade funcional da obra.

Para que seja possível verificar a resistência de materiais residuais na confecção de blocos de concreto, garantindo a viabilidade estrutural do material, é preciso apresentar um comparativo baseado em estudos práticos e ensaios realizados por alguns autores e além de destacar Silva (2019) que norteou seus estudos no elemento vidro temperado, é relevante também destacar Zanandréa (2018) que analisou a resistência de blocos de concreto confeccionados com a adição de um conjunto de elementos, descritos na Figura 2.

No ensaio de compressão simples realizado por Zanandréa (2018), norteado na NBR 9781/13, os resultados revelaram como melhor traço com o agregado miúdo reaproveitado um total de $25 \%$, chegando-se até a 34,45 MPa ao término dos 28 dias de idade, o que se assemelha aos resultados dos estudos de Hood (2006), que a partir de pesquisas isonômicas às de Zanandréa (2018), verificou um traço total de 26,67 $\mathrm{Mpa}$, chegando-se à conclusão de que esses blocos de concreto reciclados devem ser destinados a áreas de 


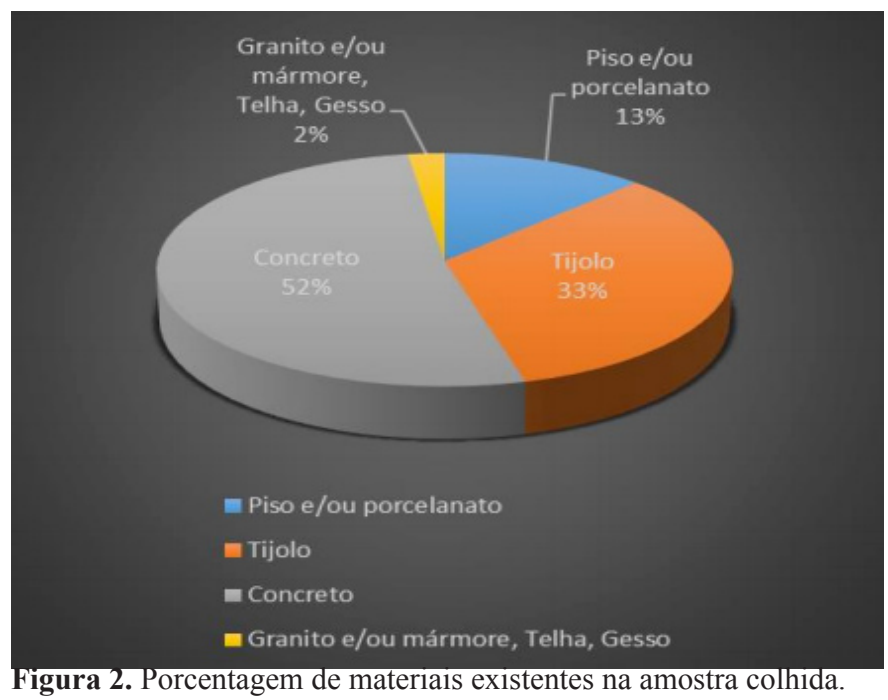

Fonte: Zanandréa (2018).

menor tráfego, com destaque a áreas de calçadas.

Ao realizar um ensaio prático, Zucca et al. (2018) verificou que os resíduos sólidos oriundos da construção civil podem e devem ser reutilizados, pois essa ação, além de reduzir significativamente o volume de entulho destinado a aterros e lixões, geralmente, irregulares, contribuir para reduzir o impacto ambiental, ainda beneficia toda a obra, principalmente quando reaproveitar esses elementos para produção de blocos de concreto, pois se trata de uma alternativa viável, de baixo custo, recomendada tecnicamente para vedação, levando a resultados muito satisfatórios.

Infelizmente, apesar da importância do reaproveitamento dos resíduos sólidos da construção civil representar um recurso viável e de baixo custo, que gera inúmeros benefícios não só ambientais, mas também financeiros para a obra, ainda é muito insignificante o número de empresas que investem nessa área, valendo destacar ainda o que dispõe Zucca et al. (2018, p. 1383):

Como estudos para aproveitamento de Resíduos de Construção Civil (RCC) empregados na forma de agregados graúdos ainda são limitados no país, e que são poucas usinas ou empresas que realizam o beneficiamento, estudos que envolvam este tema vem de encontro à necessidade de desenvolvimento civil sustentável no meio rural. Assim, pesquisar o uso de RCC como agregado graúdo como alternativa ao agregado graúdo convencional (brita), com a finalidade de confeccionar alvenaria de vedação ou blocos de concreto é uma alternativa sustentável de reuso de materiais que justifica o presente estudo.

É notável que são escassos ainda os estudos sobre a questão do reaproveitamento dos resíduos sólidos da construção civil, principalmente, para a confecção de blocos de concreto, mas gradativamente, o tema vem ganhando espaço e uma atenção maior vem sendo demandada, o que se deve ao fato de gerar benefícios ambientais e também financeiros para a obra.

Zanandréa (2018) esclarece que a simples segregação éa maneira mais comum de reaproveitamento dos resíduos sólidos da construção civil, o que reduz a retirada de recursos naturais, a partir do momento que elementos da própria obra podem ser reutilizados para fins específicos, como em substituição do agregado natural na confecção de blocos de concreto destinados a diferentes fins, inclusive, pavimentação intertravada.

Em seus estudos, Silva (2019) analisou o reaproveitamento dos resíduos sólidos da construção civil, através de estudos práticos, destacando o vidro temperado como elemento que pode ser utilizado na confecção de blocos de concreto. O autor verificou que é necessário que os materiais escolhidos para reaproveitamento atendam às exigências físicas, mecânicas e também de durabilidade exigidas por lei, para que seja possível realizar um processo de reciclagem de sucesso.

Ainda conforme Silva (2019, p. 6):

[...] tornam-se necessários estudos que visam diminuir a utilização de recursos naturais no mercado da construção civil. Para que se possa reduzir a extração de novas matérias-primas do meio ambiente na fabricação de blocos de concreto, verificou-se a viabilidade de utilizar resíduos de pó de vidro temperado processado como parte do agregado miúdo usado na fabricação dos mesmos, proporcionando uma destinação final deste material, contribuindo de forma saudável com o meio ambiente, e incentivando a prática da reciclagem com vantagens econômicas e ambientais, no mercado da construção civil. Os resultados encontrados foram satisfatórios, o que indica a sua utilização na construção civil conforme normas da ABNT NBR 6136/2016 e NBR 12118/2013 que estabelece requisitos para a fabricação dos blocos de concreto. 
Nota-se que o vidro temperado é um dos materiais encontrados nos resíduos sólidos oriundos da construção civil que podem ser reaproveitado, gerando dessa forma, inúmeros benefícios ao meio ambiente, assim como ao processo financeiro da obra, o que revela a importância da reutilização desses materiais.

Em estudos realizados por Garcia (2018) focando a reutilização de resíduos sólidos da construção civil, verificou-se que os entulhos gerados precisam ser reciclados, pois apresentam muitos fragmentos que servem como matéria prima para a fabricação de outros produtos que podem ser utilizados nas obras, inclusive blocos de concreto, o que se revela um instrumento de sucesso nos canteiros de obra, pois gera economia e ainda reduz o impacto ambiental.

\section{Considerações finais}

A partir da revisão de literatura realizada, o objetivo proposto para o presente estudo foi alcançado, uma vez, foi apresentada uma reflexão sobre o reaproveitamento de resíduos sólidos para confecção de blocos de concreto, sendo verificado que esse recurso é essencial para promover maior preservação ambiental, além de gerar redução dos custos no canteiro de obras.

É possível inferir que ao longo do estudo demonstrou-se que os resíduos sólidos são materiais gerados a partir das atividades humanas, sendo a construção civil uma das principais responsáveis pelo elevado volume desses elementos, gerando prejuízos incalculáveis ao meio ambiente, em especial, pela destinação inadequada dos mesmos.

Só na Região Sudeste há uma média de 51.582 toneladas de resíduos sólidos produzidos diariamente, o que representam dados preocupantes e demandam reflexões acerca das medidas mais adequadas a serem tomadas para reverter esse quadro, que gera inúmeros prejuízos ao meio ambiente, além de encarecer as obras realizadas na construção civil em face do desperdício de materiais que poderiam ser reaproveitados.

É importante considerar ainda que o gerenciamento dos resíduos produzidos pela construção civil envolve uma série de ações planejadas, que tem como objetivo minimizar o volume desses materiais, conscientizando as pessoas sobre a necessidade de preservação ambiental e para que seja possível melhor promover esse instrumento, é relevante que ocorra uma separação dos diferentes tipos de resíduos, conforme suas classificações, visando identificar aqueles que podem ser reutilizados.

Conclui-se ainda que uma alternativa viável e sustentável que pode ser adotada para o reaproveitamento dos resíduos sólidos é a confecção de blocos de concreto, uma vez que a matéria prima dos mesmos é retirada da própria obra, reduzindo significativamente o volume de resíduos sólidos, e tendo como resultado um produto eficiente e que pode gerar economia nos custos da obra.

\section{Referências}

Araújo, Andréa., Gouveia, Luis Borges. (2018). Pressupostos sobre a pesquisa cientifica e os testes piloto. Portugal: Universidade Fernando Pessoa.

Brasil. (2002). Resolução no 307 de 5 de julho de 2002. Estabelece diretrizes, critérios e procedimentos para a gestão dos resíduos da construção civil. Brasília: Conselho Nacional do Meio Ambiente. Ministério do Meio Ambiente.

Brasil. (2010). Politica Nacional de Resíduos Sólidos. Brasília: Ministério do Meio Ambiente.

Dantas, Bárbara de Oliveira Lage. (2018). Reutilização de resíduos sólidos da construção civil e de pneus inservíveis na pavimentação. Trabalho de Conclusão de Curso, Universidade Federal da Paraíba, João Pessoa, Pb, Brasil.

Fernandes, Graziella Quint (2018). Resíduos de construção e demolição: uma abordagem do assunto e a situação do município de Florianópolis. Trabalho de Conclusão de Curso (Especialização), Universidade do Sul de Santa Catarina, Florianópolis, SC, Brasil.

Garcia, Gabriela Di Mateos (2018). Uso de resíduos de concreto na fabricação de pavimento asfáltico. Dissertação de Mestrado, Universidade Federal de Mato Grosso, Mato Grosso, Brasil.

Hood, Rogério (2006). Análise da viabilidade técnica da utilização de resíduos de construção e demolição como agregado miúdo reciclado na confecção de blocos de concreto para pavimentação. Dissertação de Mestrado, UFRGS, Porto Alegre, Rio Grande do Sul, Brasil.

Leite, Izabella Caroline de Almeida., Damasceno, João Luís Corrêa., Reis, Alexandre Magrineli dos., Alvim, Marina. (2018). Gestão de resíduos na construção civil: um estudo em Belo Horizonte e Região Metropolitana. REEC - Revista Eletrônica de Engenharia Civil Vol. $14-n^{\circ} 1$.

Marcetti, Ana Carolina de Angeli., Lírio, Helene de Oliveira. (2018). Análise do cenário de gestão e geração de resíduos sólidos da construção civil do Municipio de Aracruz. Aracruz: Faculdades Integradas de Aracruz.

Medrade, Maicon Ortiz. (2018). Reutilização dos resíduos da construção civil e demolição - RCD. Campo Grande: UNIDERP.

Pontes, Alada Marcela Souza de Albuquerque., Araújo, Elisa Rodrigues. (2018). Análise da utilização de borracha de pneu como redução de resíduos sólidos para produção de blocos de concreto. Maceió: Centro Universitário CESMAC.

Silva, Fabrício Carvalho da. (2018). Mapeamento de tecnologias associadas ao reaproveitamento de resíduos sólidos e reciclagem de materiais utilizados no setor da construção civil brasileira. ISTI - Aracaju/SE - 21 a 23/09/ 2018. Vol. $3 / n .1 /$ p.048-057.

Silva, Maria Naira Gomes. (2019). Reaproveitamento de resíduos de vidro temperado para produção de blocos de concreto tipo vedação. Goiânia: Centro Universitário de Goiás.

Zanandréa, Kátia. (2018). Análise da resistência à compressão simples do pavimento intertravado com substituição parcial de agregados por $R C D$. Passo Fundo: Faculdade IMED.

Zucca, Rafael., Neto, José Gabriel Vieira., Santos, Rodrigo Couto., Lovatto, Juliano., Costa, Matheus Viero. (2018). Uso de resíduos de construção como agregado graúdo destinado à confecção de blocos para alvenaria de vedação. ENCICLOPÉDIA BIOSFERA, Centro Científico Conhecer Goiânia, v.15 n.27. 